\title{
De sustentados a sustentadores: El rol de las personas mayores en la familia durante la crisis económica
}

\author{
Ana Pérez Albarracín e Inmaculada Montero García. Universidad de Granada \\ Recepción: 14 de marzo de 2016 | Revisión: 14 de marzo de 2016 | Aceptado: 15 marzo de 2016 \\ Correspondencia: imontero@ugr.es \\ Citar: Perez-Albarracin, A. y Montero-Garcia, I. (2016). De sustentados a sustentadores: El rol de las personas mayores en la familia \\ durante la crisis económica. ReiDoCrea, 5, 40-55.
}

\begin{abstract}
Resumen: El presente trabajo pretende realizar una descripción del apoyo que prestan las personas mayores a sus familias desde el inicio de la crisis. Como consecuencia de la crisis económica, la situación social se está invirtiendo, de manera que los mayores están pasando de ser "sustentados" a "sustentadores". Los objetivos van dirigidos a constatar si, efectivamente, durante la crisis económica ha habido un aumento en la prestación de su apoyo; Identificar, en caso positivo, en qué aspectos se ha incrementado el apoyo y, lo que realmente constituye un aporte sustancial, describir cómo perciben las personas mayores dicho incremento. Como instrumento de recogida de datos se ha elaborado un cuestionario y como población se han escogido tres grupos de sujetos a partir de diferentes contextos. Los resultados obtenidos permiten constatar un aumento en la prestación del apoyo. No obstante, aunque con variaciones significativas según el contexto considerado, valoran positivamente el incremento de la ayuda facilitada.
\end{abstract}

Palabras clave: Personas Mayores | Familia

From supported to supporters: The role of seniors in the family during the economic crisis

\begin{abstract}
This paper aims to provide a description of the support provided by seniors to their families since the beginning of the crisis. As a result of the economic crisis, the social situation is being reversed, so that seniors are going from being "supported" to "supporters". The objectives are aimed at determining if, in fact, there has been an increase in the provision of support from this group during the economic crisis; If so, to identify, in which aspects the support has increased and, as a substantial contribution, to describe how seniors perceive this increase. A questionnaire was developed as an instrument for data collection, and three groups of participants were chosen from different contexts. The results indicate that there was an increase in the provision of support. However, even with significant variations depending on the context in question, they value positively the increase in assistance provided.
\end{abstract}

Keywords: Seniors | Family

\section{Introducción}

La mayoría de los autores que han indagado en los orígenes y las causas de la crisis económica española, sitúan sus inicios entre los últimos meses del año 2007 y los primeros de 2008 (Andreu, 2007; Colom, 2012; Fernández, 2013; Villar, 2009). Según Colom (2012), se considera dicha crisis desde mediados de 2008: "la economía española entró técnicamente en recesión entre el segundo y tercer trimestre de 2008 al presentar durante dos trimestres consecutivos tasas de crecimiento negativas". Siguiendo al mismo autor, las causas del deterioro económico son concretamente dos: la burbuja inmobiliaria y la crisis financiera mundial. La etapa de crecimiento de la economía española tuvo lugar en 1997 coincidiendo con el aumento de demanda y por tanto, de empleo en el sector de la construcción, cuando un $13,25 \%$ de la población ocupada trabajaba en él. Debido a que se seguían construyendo viviendas que no tenían comprador, este sector cayó, dejando desempleadas a un gran número de personas. Por otro lado, la crisis económica española se vio acompañada por una crisis mundial, iniciada en Estados Unidos en los primeros meses de 2007, después de haber concedido durante años hipotecas a particulares sin apenas exigencias o avales. Esta situación derivó en un aumento de la morosidad y, en consecuencia, del número de bancos en quiebra. La actual situación española es por tanto, según Villar (2009), la conjunción de dos crisis, la española y la internacional. 
Pero la crisis económica no ha afectado únicamente a bancos y grandes empresas sino que son los ciudadanos "de a pie" los que observan cómo menguan día a día sus ingresos. Como apunta Cruces (2013):

Los progresivos recortes en derechos de carácter social, el proceso de devaluación interna en el que estamos inmersos, pero también la "reconfiguración" de la Ley de Atención a las personas en situación de dependencia, el copago sanitario, la exclusión de más de 450 medicamentos de uso común de las prestaciones de la sanidad pública, la reducción de becas y ayudas al estudio incluso en los tramos de escolarización obligatoria, o el endurecimiento de las condiciones para percibir el subsidio una vez finaliza la prestación contributiva por desempleo, están configurando una situación cada vez más insostenible con menor volumen de ingreso de las familias y un aumento del gasto en servicios de carácter social.

A pesar de que son muchos los cambios socioeconómicos que están atravesando las familias españolas, podría considerarse que algunos grupos de población se han visto favorecidos por la situación actual. Así, las personas mayores, han visto reducida su pobreza relativa como consecuencia de un empobrecimiento generalizado de la población, si bien es cierto, como explica el informe de la Fundación FOESSA (2014), que aunque bajan los umbrales de pobreza, los índices no dejan de aumentar. También cabe considerar que la tercera parte de las pensiones de jubilación no superaron los 700 euros en 2011 y que entre 2008 y 2012 el 11 por ciento de las personas desahuciadas eran pensionistas (Cruces, 2013). Asimismo, a pesar de que actualmente muchos de nuestros mayores viven un envejecimiento activo como participación continua en las cuestiones sociales, económicas, culturales, espirituales y cívicas (Montero y Muñoz, 2013; Fernández, 2014), alejados de la innecesaria alarma social de la dependencia, siguen siendo los principales afectados en los recortes de carácter social que se acaban de mencionar, tales como la Ley de Dependencia o el copago sanitario. Por lo tanto, la situación socioeconómica de estas personas es más delicada de lo que parece. Los hogares con pensionistas estarian siendo en esta crisis los menos ayudados. La razón de ello no se debería a una falta de solidaridad con estos núcleos sino, más bien, que las pensiones están siendo un contrapeso fundamental a la pérdida de ingresos. Los mayores de 65 años son el colectivo que más ayuda ofrece a la familia en calidad de benefactores, mostrando así un aumento importante del año 2007 al 2013. Por contra, el impacto de la crisis ha hecho precisamente que sean los jóvenes menores de 30 años el único grupo que ha crecido entre los receptores de ayuda entre 2007 y 2009 , representando del $21,8 \%$ al 26,6\% (Martínez, 2014).

Parece evidente que, además de ver cómo la crisis les afecta directamente, las personas mayores se ven en la obligación de colaborar con aquellos familiares en los que la crisis también va haciendo mella. Cruz Roja (2012), califica la aportación de los mayores como un pilar imprescindible de la contención social. Es cierto que el papel de las personas mayores en las familias viene cobrando relevancia en las últimas décadas, desde que la mujer se incorporó al mundo laboral y los abuelos y abuelas se han convertido en cuidadores principales de sus nietos. Este aumento del apoyo que prestan los mayores respecto al cuidado de los nietos ha sido explicitado tanto por autores nacionales (IMSERSO, 2010; Ortega, 2007; Tobío, 2010) como internacionales (Baker, 2008; Kirby, 2013; Vandell, 2003). De hecho, según un Informe del IMSERSO (2012) en Europa más del $40 \%$ de los abuelos se encargan del cuidado de sus nietos. Actualmente, nos encontramos con una situación en la que, a la contribución que ya venían haciendo las personas mayores, se suma el papel de sustentadores principales de un porcentaje elevado de familias españolas. En el tercer trimestre de 2012, el 26\% por ciento de los hogares tenían como persona de referencia una persona jubilada o con una pensión distinta a la de jubilación y en un 
$27,3 \%$ existía un mayor de 64 como sustentador principal (INE, 2012a). Es decir, como consecuencia de la crisis económica, la situación social se está invirtiendo, de manera que los mayores están pasando de ser "sustentados" a "sustentadores" (Cruces, 2013, p.33). O, lo que es lo mismo, de ser considerados personas pasivas, sin recursos y susceptibles de recibir ayuda, a convertirse en agentes dinámicos, participando de forma directa en la economía y otras responsabilidades que tienen que ver con el apoyo a sus familias.

De hecho, como exponen Hidalgo, Calderón y Pérez (2008), ya desde el comienzo de la crisis proliferaban los hogares sustentados por mayores y además estaban en riesgo:

El envejecimiento de la población y el aumento de la esperanza de vida han hecho incrementarse el número de hogares cuyo sustentador principal es una persona mayor de 65 años, y cuyas peculiaridades respecto a los hogares sustentados por personas de otros grupos de edad, aumentan la propensión de los primeros para caer en la pobreza (p.12). Estudios muy recientes han mostrado cómo durante los años 2007-2013 han aumentado aquellos hogares donde existe la presencia de personas mayores de 65 años como benefactores en 9,6 puntos (34,1\%-43,7\%), poniendo de nuevo de manifiesto que las pensiones han incrementado su potencial integrador. No obstante, en relación a este aspecto cabría matizar que muchas de las cuantías de estas prestaciones son muy bajas, por lo que aunque se ofrezca ayuda, los efectos pueden tener importantes costes en los hogares pensionistas (Martínez, 2014, 17).

En cuanto a la procedencia de estas ayudas, atendiendo a la principal fuente de ingresos, el $47,51 \%$ se obtiene del trabajo por cuenta ajena, y casi el $40 \%$ de pensiones, subsidios por desempleo, y otras prestaciones y ayudas públicas (López, 2013). Según el estudio "Crisis y fractura social" de la Fundación La Caixa (2012), en muchos hogares donde los ingresos se han visto reducidos por el impacto de la crisis, las pensiones, aunque sean de baja cuantía, se convierten en un recurso más estable que las rentas del trabajo. Lo cierto es que, como se expresa en la Tabla 1, la situación ha cambiado de forma radical, de tal manera que, de 2006 a 2011, los hogares sustentados por mayores de 65 años han pasado de ser los que realizaban un gasto menor a convertirse en los que mayor gasto por persona efectúan (INE, 2012b). Según Salvetti y Llombart (2015), 2 de cada 10 abuelos destinan un $40 \%$ o más de sus ingresos mensuales a ayudarles y un $10 \%$ de los entrevistados en su estudio se había visto en la necesidad de vender algún bien de su propiedad para ayudar a sus hijos / nietos. La generosidad de los mayores españoles ha ido en aumento durante la crisis y destaca sobre otros países europeos a pesar de que perciben ingresos relativamente más bajos que el resto de la Unión Europea (Pérez, Abellán y Pujol, 2013).

\begin{tabular}{|c|c|c|c|c|c|c|}
\hline \multicolumn{7}{|c|}{ Tabla 1: Gasto medio por persona según edad del sustentador principal del hogar } \\
\hline Edad del sustentador principal & Año 2006 & Año 2007 & Año 2008 & Año 2009 & Año 2010 & Año 2011 \\
\hline TOTAL & 11.055 & 11.673 & 11.801 & 11.365 & 11.161 & 11.137 \\
\hline Entre 16 y 29 años & 11.814 & 12.749 & 11.838 & 10.656 & 10.350 & 10.345 \\
\hline Entre 30 y 44 años & 10.789 & 11.318 & 10.991 & 10.407 & 10.219 & 10.050 \\
\hline Entre 45 y 64 años & 11.597 & 12.135 & 12.564 & 12.111 & 11.743 & 11.651 \\
\hline 65 y más años & 10.157 & 11.048 & 11.681 & 11.752 & 11.789 & 12.093 \\
\hline
\end{tabular}

En efecto, según investigaciones muy recientes (TFW, 2014), en los hogares donde conviven los abuelos existe un incremento importante de aquellos que colaboran de 
manera económica al sustento de la familia, pasando del 46,8 en el año 2013 al 65,2 en el 2014. De ahí nuestra preocupación por intentar destacar, más allá de las cifras y la representación social que existe acerca de las personas mayores en el impacto de la crisis, la propia imagen que sienten y manifiestan acerca de su situación en la actual coyuntura socio-económica.

\section{Objetivos}

Existe un gran número de publicaciones relacionadas con el apoyo que se les presta a las personas mayores tanto por parte de sus familiares como de instituciones diversas (Campo, 2000; Guzmán, 2003, Yangua, 2000) o incluso centradas en los efectos que conlleva ejercer de cuidador de una persona mayor (IMSERSO, 2007; Rodríguez, 2013). Sin embargo, a pesar de que la bibliografía existente también contempla a los mayores como cuidadores, especialmente de sus nietos, las publicaciones que se centran en las personas mayores como sustentadoras de la familia son escasas. La finalidad principal con esta investigación, es analizar si, a raíz de la crisis, se ha incrementado el apoyo que prestan los mayores a su familia y, en su caso, cómo es percibido por ellos. Dada la dualidad de los sustentos que prestan los mayores (de cuidado y económicos), utilizaremos esta distinción para analizar los aspectos en que se incrementa su apoyo.

A pesar de que, como se explica anteriormente, un gran porcentaje de mayores se ha visto gravemente afectado por la crisis (Cáritas, 2013; Cruz Roja, 2012), determinadas personas disponen de herramientas para hacer frente a estos episodios de forma más llevadera que el resto de la población. Un ejemplo de ello son las personas que participan en actividades de educación en el tiempo libre, ya que posibilitan, además del aprendizaje, la integración y la participación social (Alfageme, 2007; Calero, Fernández y Roa, 2009; Guerrero, 2012; Villar, 2010). Es por ello que este trabajo se centrará en tres grupos, dos de ellos involucrados en actividades socio-educativas (alumnos del Aula Permanente de Formación Abierta de la Universidad de Granada y mayores participantes en centros de día o centros de participación activa) y otro conjunto de mayores encuestados al azar en un centro de salud. De este modo se pretenden establecer comparaciones entre los tres contextos.

Dicho esto, los objetivos que finalmente se pretenden lograr con esta investigación son los siguientes:

1. Constatar si, a partir de la crisis económica, ha habido un aumento en la prestación de apoyo por parte de las personas mayores a sus familias.

2. En caso positivo, identificar en qué aspectos se ha incrementado el apoyo.

3. Describir, en su caso, cómo perciben las personas mayores este incremento.

\section{Método}

\section{Participantes}

El tipo de muestreo ha sido intencional, atendiendo a la facilidad para acceder a los entornos elegidos y tomando a su vez a personas pertenecientes a tres contextos con características muy distintas y precisas al objeto de contrastar e inferir resultados. En esta investigación han participado 87 personas mayores de 55 años, que han decidido colaborar de manera voluntaria y anónima. A pesar de que a los 55 años no se considera que una persona es aún "persona mayor", se ha incluido esta franja de edad en tanto que al Aula Permanente de Formación Abierta de la Universidad de Granada se accede regularmente a partir de los 55 años y el análisis de este contexto es 
relevante para los propósitos de la investigación. Siendo tres los grupos encuestados, se dispuso de $\mathrm{N}=42$ participantes alumnos del Aula Permanente de Formación Abierta (APFA) $(39 \%), \mathrm{N}=24$ participantes asistentes a centros de participación activa $(23 \%)$ y $\mathrm{N}=41$ encuestados en el centro de salud (38\%) (véase gráfico 1).

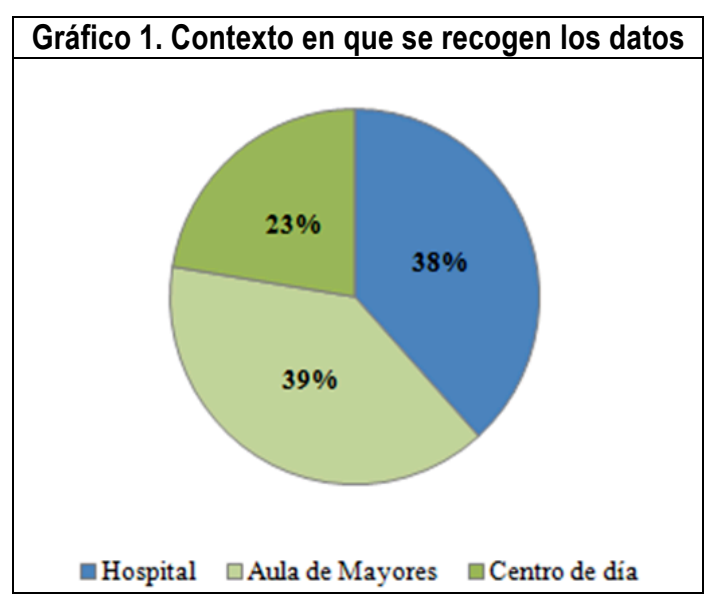

Respecto a la edad, el $41 \%$ de los participantes tenía entre 55 y 64 años, el $35 \%$ entre 65 y 74 , el $18 \%$ entre $75-84$ años y un $6 \%$ fueron mayores de 85 (véase gráfico 2).

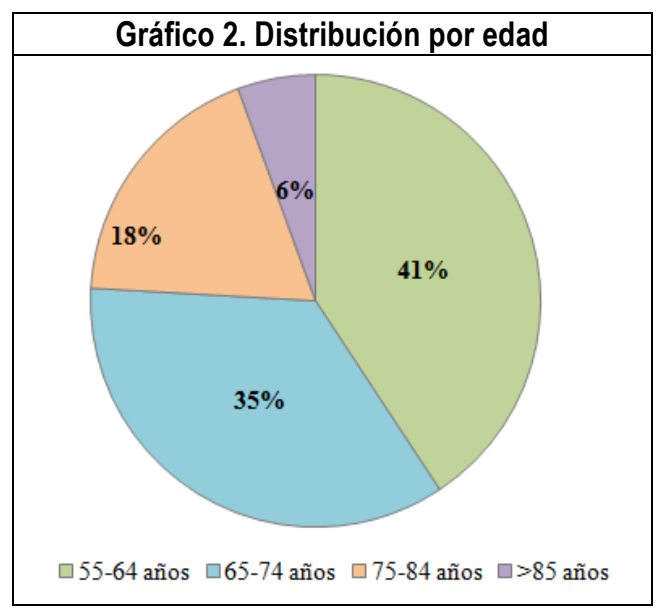

En cuanto a la distribución por sexo, participaron un $67 \%$ de mujeres y un $33 \%$ de hombres (véase gráfico 3).

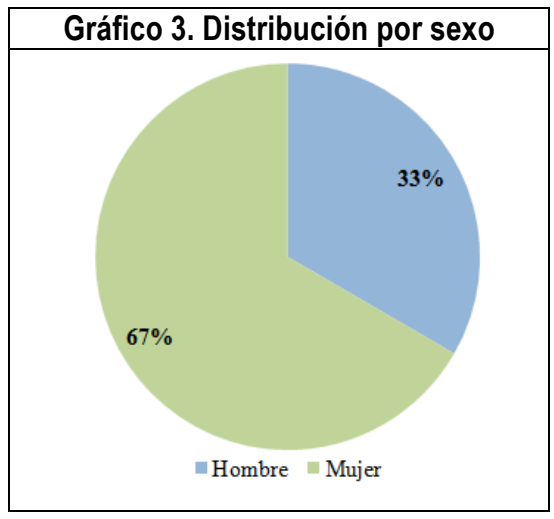


Como se observa en el gráfico 4, la mayoría de las personas que han respondido al cuestionario afirmaba residir en su propia casa (91\%). Solo un $9 \%$ vivía en casa de sus hijos o en otros lugares.

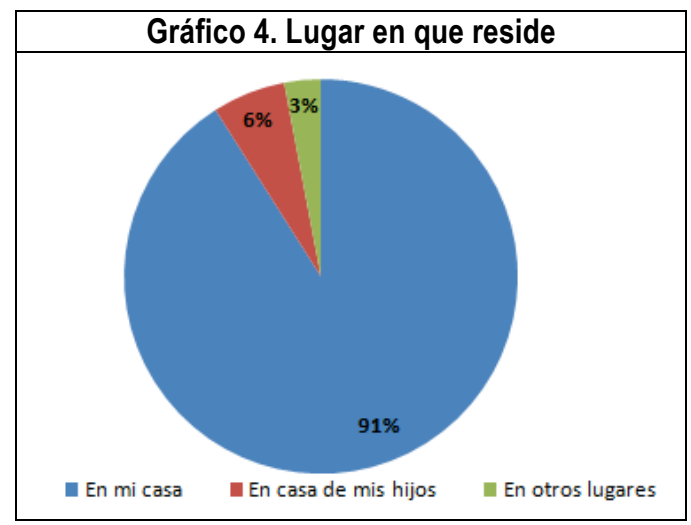

Una parte importante de ellos vivía con su pareja (36\%), seguido de las personas que viven solas $(25 \%)$, las que viven con sus hijos $(22 \%)$ y las que viven tanto con sus hijos como con su pareja (11\%). El resto de las personas que han respondido a esta pregunta viven con otros familiares (véase gráfico 5).

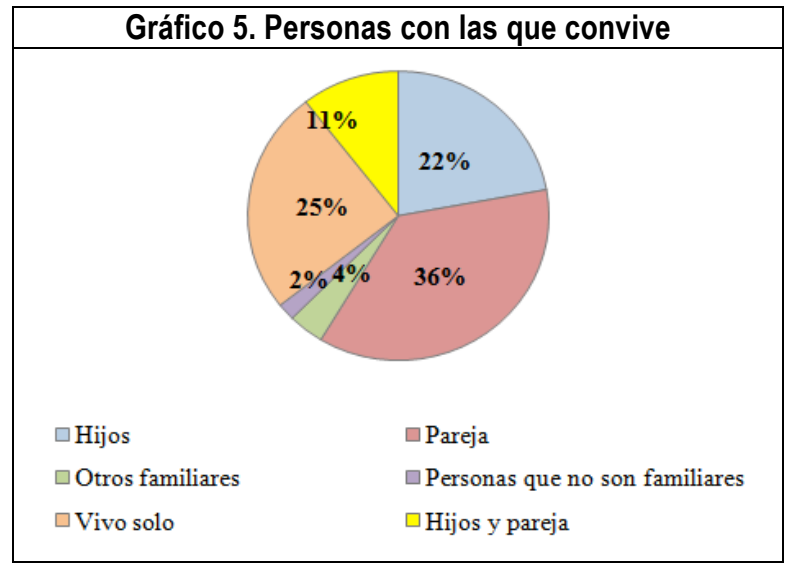

En lo que se refiere al estado civil, más de la mitad de la muestra $(51 \%)$ está casada, aunque un porcentaje importante está viudo/a (27\%) o soltero/a (13\%). El resto están divorciados o separados (véase gráfico 6).

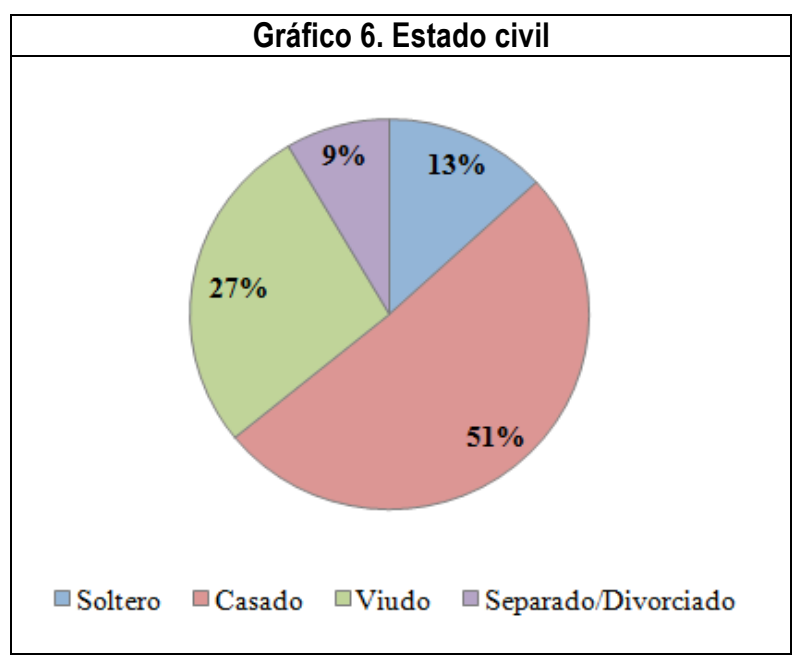


En el gráfico 7 se puede observar el número de hijos de los encuestados, siendo un gran porcentaje de personas las que afirman tener dos hijos (24\%).

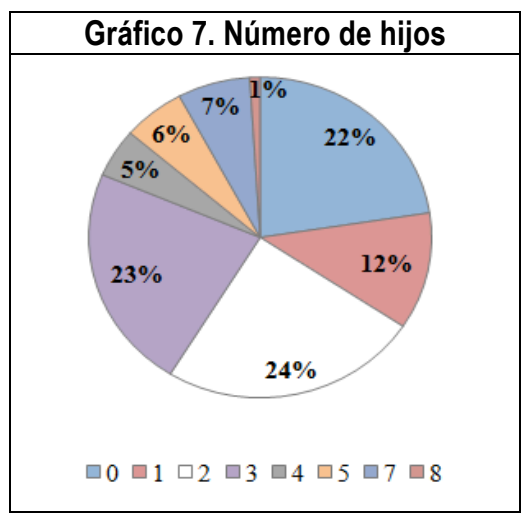

Aproximadamente la mitad de la muestra tiene un alto nivel socioeconómico, siendo un $37 \%$ las personas que perciben más de 1200 euros al mes (véase gráfico 8). Destaca la diferencia económica en las franjas más elevadas, donde el Aula Permanente tiene ingresos superiores a $1200 €(71 \%)$, seguido del centro de salud $(29 \%)$; ninguno de los encuestados en el centro de día dispone de tal cantidad de ingresos (véase gráfico 9).
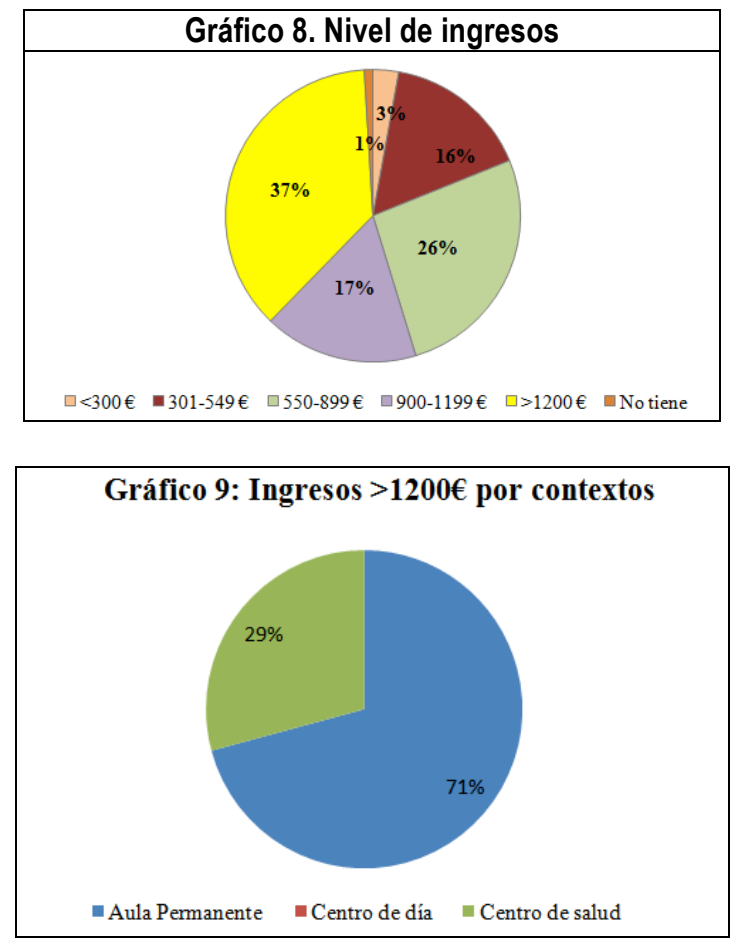

\section{Instrumentos y procedimiento}

El instrumento utilizado para lograr los objetivos propuestos ha sido el cuestionario dado que permite recoger datos de una muestra más amplia en menor tiempo que otros instrumentos. Además facilita la valoración de los datos en tanto se obtienen datos cuantificables. No obstante, se han incluido preguntas abiertas para contrastar e interpretar los resultados cuantitativos.

El cuestionario utilizado, como se puede observar en el Anexo 1, se divide en dos partes: una primera en la que se contemplan variables sociodemográficas y una segunda con las variables objeto de estudio. 
En la primera parte, las variables que nos interesan para caracterizar la muestra son la edad, el sexo, el lugar en que reside, las personas con las que convive, el número de hijos y el nivel de ingresos mensuales. La mayoría de estas preguntas son de respuesta dicotómica, sin embargo, la pregunta "personas con las que convive" es de respuesta múltiple. También es necesario especificar que en la cuestión "lugar en que reside" se ha considerado la opción "otro", dejando un ítem abierto en el que los encuestados pudieran exponer otras posibles alternativas que no hubieran sido contempladas a la hora del diseño. Cabe mencionar que los intervalos de ingresos propuestos están basados en un informe reciente de Cruz Roja (2012) que se relaciona con el objeto de estudio de esta investigación. Además, en esta primera parte se contemplan también otro tipo de variables consideradas de interés para el estudio: el tipo y frecuencia del contacto con su familia y las actividades en que participa. Esta segunda pregunta también es de respuesta múltiple e incluye la respuesta abierta "otros". En cuanto a las actividades de ocio que aparecen en el instrumento están basadas en el informe del IMSERSO (2012), en que se indica que son éstas las realizadas con mayor frecuencia por las personas mayores.

La segunda parte comienza planteando dos preguntas que nos permiten obtener un idea previa de su opinión sobre el hecho de prestar apoyo y que responden al objetivo que hace referencia a la ayuda que ofrecen así como a la percepción que los mayores tienen acerca de este hecho: “¿Presta ayuda y apoyo a las personas de su entorno cuando lo necesitan?" "¿Es importante para usted ayudar y preocuparse por el bienestar de las personas que tiene alrededor?". Seguidamente se presentan tres preguntas centradas en los objetivos que se pretenden investigar: si ha aumentado el apoyo que prestan a sus familias desde que comenzó la crisis, en qué aspectos creen que se ha incrementado dicho apoyo y cómo se sienten respecto a ese incremento.

La primera, “¿Considera que a partir de la crisis económica ha tenido que prestar más apoyo a su familia?" es una pregunta de respuesta dicotómica que nos permite determinar si efectivamente se ha incrementado o no el apoyo. La segunda "Marque en qué aspectos le presta más apoyo", es una pregunta de respuesta múltiple donde aparecen 11 ítems referentes a los dos tipos de apoyo que se pretenden investigar: cuatro ítems sobre apoyo de cuidado, seis sobre apoyo económico y un último denominado "otros" que deja la pregunta abierta a otras respuestas. La tercera variable en la que se pretende indagar, "Indique cómo percibe este cambio" también presenta 9 ítems, cuatro formulados en positivo, cuatro en negativo y un último de respuesta abierta.

Tras discutir el diseño en profundidad, consideramos necesario someterlo a un "juicio de expertos" para determinar su validez, así como la realización de una pasación piloto que determinara si el instrumento se ajustaba a las características de los participantes respondiendo a criterios de validez metodológica.

La dificultad principal a la hora de elaborar el cuestionario se hallaba en adecuar los ítems a la población escogida, tanto a nivel de comprensión como en la presentación de las preguntas, de manera que no se sintieran incómodas al contestar. Tras el juicio de expertos, en el que participaron profesores de las áreas de psicología, métodos en investigación y teoría de la educación, se concluyó que el cuestionario cumplía los criterios de validación y podía lograr los objetivos propuestos, por lo que se pasó a realizar una prueba piloto. En ella se modificaron algunas preguntas que presentaban dificultades, esencialmente de respuesta abierta, de forma que se optó por convertirlas en cerradas evitando así que indujeran a error. 


\section{Análisis de datos}

Para analizar los datos obtenidos se ha utilizado el programa de análisis estadístico SPSS 20. También, con el propósito de una mejor presentación de los resultados, se utilizó la Hoja de cálculo Excel destinado a realizar los gráficos y las tablas. El análisis consiste en realizar un análisis porcentual de cada una de las variables objeto de estudio.

\section{Resultados}

A continuación se presenta el análisis porcentual obtenido tras el tratamiento de los datos.

Un $61,8 \%$ de los encuestados afirma que, a partir de la crisis económica, ha tenido que prestar más apoyo a su familia.

El apoyo de cuidado se incrementa en torno a un $20 \%$ (véase tabla 2). No obstante, cada contexto se centra en mayor medida en uno de los apoyos: el Aula Permanente en los hijos no emancipados $(29,2 \%)$, el centro de participación activa en el cuidado de los nietos $(29,4 \%)$ y los mayores del centro de salud en elaborar las dietas de sus familiares $(32,3 \%)$ (véase tabla 3 ).

\begin{tabular}{|l|r|l|}
\hline \multicolumn{3}{|c|}{ Tabla 2. Aspectos en los que se incrementa el apoyo de cuidado } \\
\hline & \multicolumn{1}{|c|}{ Sí } & No \\
\hline Cuido de mis nietos diariamente & $13-18,1 \%$ & $59-81,9 \%$ \\
\hline Mis hijos (y/o nietos) comen en mi casa a diario & $15-20,8 \%$ & $57-79,2 \%$ \\
\hline Colaboro en las tareas del hogar de mis hijos & $8-11,1 \%$ & $64-88,9 \%$ \\
\hline Mis hijos todavía no han podido independizarse y viven conmigo & $13-18,1 \%$ & $59-81,9 \%$ \\
\hline
\end{tabular}

Tabla 3. Aspectos en los que se incrementa el apoyo de cuidado por contextos

\begin{tabular}{|c|c|c|c|c|c|c|}
\hline & \multicolumn{2}{|l|}{ APFA } & \multicolumn{2}{|c|}{ Centro de día } & \multicolumn{2}{|c|}{ Hospital } \\
\hline & Sí & No & Sí & No & Sí & No \\
\hline \multirow[t]{2}{*}{ Cuido de mis nietos diariamente } & 3 & 21 & 5 & 12 & 5 & 26 \\
\hline & $12,5 \%$ & $87,5 \%$ & $29,4 \%$ & $70,6 \%$ & $16,1 \%$ & $83,9 \%$ \\
\hline \multirow[t]{2}{*}{ Mis hijos (y/o nietos) comen en mi casa a diario } & 4 & 20 & 1 & 16 & 10 & 21 \\
\hline & $16,7 \%$ & $83,3 \%$ & $5,9 \%$ & $94,1 \%$ & $32.3 \%$ & $67,7 \%$ \\
\hline \multirow[t]{2}{*}{ Colaboro en las tareas del hogar de mis hijos } & 0 & 24 & 3 & 14 & 5 & 26 \\
\hline & $0 \%$ & $100 \%$ & $17,6 \%$ & $82,4 \%$ & $16,1 \%$ & $83,9 \%$ \\
\hline \multirow[t]{2}{*}{ Mis hijos todavía no han podido independizarse y viven conmigo } & 7 & 17 & 1 & 16 & 5 & 26 \\
\hline & $29,2 \%$ & $70,8 \%$ & $5,9 \%$ & $94,1 \%$ & $16,1 \%$ & $83,9 \%$ \\
\hline
\end{tabular}

Como se aprecia en la tabla 4, si bien los encuestados no se consideran los sustentadores principales de la familia (solo un $16,7 \%)$, un porcentaje elevado $(58,3 \%$ ) afirma que presta apoyo económico de manera regular.

\begin{tabular}{|lrc|}
\hline \multicolumn{3}{|c|}{ Tabla 4. Aspectos en los que se incrementa el apoyo económico } \\
\hline \multicolumn{3}{|c|}{ Sí } \\
\hline Presto apoyo económico de manera regular & $42-58,3 \%$ & $30-41,7 \%$ \\
He tenido que hacer un préstamo importante a mis hijos & $11-15,3 \%$ & $61-84,7 \%$ \\
Mis hijos (y nietos, si es el caso) han vuelto a vivir conmigo por motivos económicos & $3-4,2 \%$ & $69-95,8 \%$ \\
Mis hijos residen en una vivienda que es de mi propiedad & $10-13,9 \%$ & $62-86,1 \%$ \\
He avalado a mis hijos en el préstamo que han solicitado & $12-16,7 \%$ & $60-83,3 \%$ \\
Soy el sustentador principal de la familia & $12-16,7 \%$ & $60-83,3 \%$ \\
\hline
\end{tabular}


El incremento en el apoyo económico de manera regular se puede observar en los tres contextos, siendo un $54,2 \%$ en el Aula Permanente, un $58,8 \%$ en el centro de participación activa y un $61,3 \%$ en el centro de salud (véase tabla 5).

\begin{tabular}{|c|c|c|c|c|c|c|}
\hline & \multicolumn{2}{|c|}{ APFA } & \multicolumn{2}{|c|}{ Centro de participación activa } & \multicolumn{2}{|c|}{ Hospital } \\
\hline & Sí & No & Sí & No & Sí & No \\
\hline \multirow[t]{2}{*}{ Presto apoyo económico de manera regular } & 13 & 11 & 10 & 7 & 19 & 12 \\
\hline & $54,2 \%$ & $45,8 \%$ & $58,8 \%$ & $41,2 \%$ & $61,3 \%$ & $38,7 \%$ \\
\hline \multirow{2}{*}{$\begin{array}{l}\text { He tenido que hacer un préstamo importante a } \\
\text { mis hijos }\end{array}$} & 2 & 22 & 4 & 13 & 5 & 26 \\
\hline & $8,3 \%$ & $91,7 \%$ & $23,5 \%$ & $76,5 \%$ & $16,1 \%$ & $83,9 \%$ \\
\hline \multirow{2}{*}{$\begin{array}{l}\text { Mis hijos (y nietos, si es el caso) han vuelto a } \\
\text { vivir conmigo por motivos económicos }\end{array}$} & 1 & 23 & 0 & 17 & 2 & 29 \\
\hline & $4,2 \%$ & $95,8 \%$ & $0 \%$ & $100 \%$ & 6,5 & $93,5 \%$ \\
\hline \multirow{2}{*}{$\begin{array}{l}\text { Mis hijos residen en una vivienda que es de mi } \\
\text { propiedad }\end{array}$} & 5 & 19 & 3 & 14 & 2 & 29 \\
\hline & $20,8 \%$ & $79,2 \%$ & $17,6 \%$ & $82,4 \%$ & 6,5 & $93,5 \%$ \\
\hline \multirow{2}{*}{$\begin{array}{l}\text { He avalado a mis hijos en el préstamo que han } \\
\text { solicitado }\end{array}$} & 2 & 22 & 2 & 15 & 8 & 23 \\
\hline & $8,3 \%$ & $91,7 \%$ & $11,8 \%$ & $88,2 \%$ & $25,8 \%$ & $74,2 \%$ \\
\hline \multirow[t]{2}{*}{ Soy el sustentador principal de la familia } & 4 & 20 & 2 & 15 & 6 & 25 \\
\hline & $16,7 \%$ & $83,3 \%$ & $11,8 \%$ & $88,2 \%$ & $19,4 \%$ & $80,6 \%$ \\
\hline
\end{tabular}

En la tabla 6, los encuestados parecen tener una percepción positiva del cambio; en este sentido un $23,9 \%$ se siente más valorado, el $35,2 \%$ se siente más útil y el $47,9 \%$ querría ayudar más a su familia.

\begin{tabular}{|c|c|c|}
\hline \multicolumn{3}{|c|}{ Tabla 6. Percepción positiva del cambio } \\
\hline & Sí & No \\
\hline En general, me siento mucho mejor & $13-18,3 \%$ & $58-81,7 \%$ \\
\hline Me siento más útil & $25-35,2 \%$ & $46-42,6 \%$ \\
\hline Me siento más valorado/a por mi familia & $17-23,9 \%$ & $54-76,1 \%$ \\
\hline Me gustaría poder ayudar más a mi familia & $34-47,9 \%$ & $37-52,1 \%$ \\
\hline
\end{tabular}

Sin embargo, un $25,4 \%$ afirma tener menos tiempo para ellos mismos que antes, especialmente en el centro de salud, donde el porcentaje que selecciona este ítem se eleva al 33,3\% (véase tabla 7 ).

\begin{tabular}{|lcc|}
\hline \multicolumn{3}{|c|}{ Tabla 7. Ítems de percepción negativa del cambio } \\
\hline & Sí & No \\
\hline En ocasiones, siento que mi familia se aprovecha de mi ayuda & $6-8,5 \%$ & $65-91,5 \%$ \\
Tengo menos tiempo para mí que antes & $18-25,4 \%$ & $53-74,6 \%$ \\
Siento que mi familia no valora mi ayuda lo suficiente & $10-14,1 \%$ & $61-85,9 \%$ \\
Me gustaría tener más tiempo para mí & $12-16,9 \%$ & $59-83,1 \%$ \\
\hline
\end{tabular}

\section{Discusión}

Según algunos estudios (INE, 2012a; Cruces, 2013; Hidalgo et. al. 2008; Sabater, 2014), los hogares sustentados por personas mayores de 64 años se han incrementado desde el comienzo de la crisis así como también ha aumentado el gasto de los hogares sustentados por mayores (INE, 2012b; Laparra, 2012). Sin embargo, a diferencia de lo anterior, en los resultados obtenidos en nuestra investigación se advierte que sólo una mínima parte de los participantes de este estudio reconocen ser los sustentadores principales de sus familias, o al menos, no consideran su contribución como tal. Aun así, cabe resaltar el claro incremento en el apoyo económico por parte de los mayores, dado que un $58,3 \%$ de los encuestados afirman prestar apoyo económico regular a sus familias. Este porcentaje se mantiene independientemente del contexto en el que se toman los datos. Además, un $61,8 \%$ considera que a partir de la crisis económica ha tenido que prestar más apoyo en 
general a su familia. Por tanto, los datos referidos al aumento del gasto de los hogares sustentados por mayores que expone el Instituto Nacional de Estadística (2012) se corroboran en la misma dirección a través de este estudio.

Cabe mencionar que el apoyo de cuidados que diversos autores señalan desde hace años (IMSERSO, 2012; Ortega, 2007; Tobío, 2010; Vandell, 2003), no solamente se sigue produciendo sino que, aunque en menor medida que el económico, también ha aumentado con la crisis. Así, aproximadamente el $20 \%$ de la muestra (véase tabla 2) afirma que su cuidado ha aumentado en diferentes aspectos, siendo que en APFA un $29,2 \%$ responde afirmativamente al ítem "mis hijos todavía no han podido independizarse y viven conmigo", en el centro de participación activa un $29,4 \%$ al ítem "cuido de mis nietos diariamente" y en el centro de salud un $32,3 \%$ al ítem "mis hijos (y/o nietos) comen en mi casa a diario". Si bien se podría deducir que con el aumento del desempleo y por tanto, más tiempo disponible, el cuidado de los mayores debería reducirse, lo cierto es que este cuidado se intensifica en los aspectos más básicos como la alimentación y el cuidado de los nietos.

Al respecto, habiendo constatado que, efectivamente y de acuerdo con otras investigaciones señaladas, se incrementa "de facto" el apoyo de las personas mayores durante la crisis económica, cabe recordar, tal como señalábamos en el apartado introductorio, la escasez de investigaciones que profundicen en cómo perciben los mayores dicho incremento. Como aporte fundamental de esta investigación, se concluye que los mayores perciben positivamente el aumento de su apoyo, puesto que querrían ayudar más si cabe a su familia $(47,9 \%)$ y se sienten más útiles $(35,2 \%)$ y también más valorados $(23,9 \%)$.

No obstante, un $25,2 \%$ de la muestra reconoce tener menos tiempo para ellos mismos que antes. Este aspecto destaca especialmente en la muestra de encuestados en el centro de salud, siendo que un $33,3 \%$ dice tener menos tiempo que antes y un $30 \%$, querría tener más tiempo para sí mismo. Un dato interesante es que en este mismo contexto es en el que menos útiles dicen sentirse $(26,7 \%)$, frente a un 37,5 de personas que se sienten más útiles en APFA y un $47,1 \%$ en los centros de participación activa.

De este modo, la solidaridad y las redes de ayuda que se derivan de este principio, se convierten en agentes de integración y bienestar, y el papel que juegan se define, ante todo, por la capacidad que, en momentos de crisis, pueden tener determinados miembros para prevenir y compensar las situaciones de vulnerabilidad y dificultad del resto de la familia. Si bien es preciso destacar que el envejecimiento no es un fenómeno aleatorio. La persona es agente de su propio proceso de envejecimiento, y la capacidad de envejecimiento bien saludable y activo viene, en cierta medida, de las decisiones tomadas por el mismo individuo, así como del repertorio de comportamientos aprendidos durante toda la vida (Montero, García y Bedmar, 2011; Fernández Ballesteros, 2013). La familia no queda excluida de este presupuesto. Por tanto, los modelos de convivencia con abuelos y abuelas no solo tienen como finalidad garantizar, a través de la pensión, un ingreso estable en el hogar o compensar la pérdida de una vivienda, sino que todo ello constituye una estrategia que optimiza los recursos familiares y por efecto directo, tiene implicaciones personales en cada uno de los miembros pertenecientes a ese hogar. En este sentido, es preciso subrayar que las situaciones y alternativas propuestas para la convivencia y solidaridad familiar pueden tener costes derivados de la sobrecarga de obligaciones económicas o de cuidado y apoyo por parte de las personas mayores, lo cual podría llegar a afectar y debilitar las redes familiares a otros niveles (Martínez, 2014). 
Para concluir, se retoman los objetivos de la investigación, se constata que ha habido un aumento en la prestación de apoyo por parte de las personas mayores a sus familias durante la crisis. Este incremento se percibe en los dos tipos de apoyo: el apoyo de cuidado y el apoyo económico. No obstante, el aumento es especialmente notable en el apoyo económico regular. Aun así, los datos señalan que los encuestados no se perciben a sí mismos como los sustentadores principales de sus familias. Destaca el principio de solidaridad familiar, en el sentido de que dicho incremento es reconocido positivamente por la muestra, a pesar de que admiten una falta de tiempo para dedicarse a ellos mismos. En cuanto a los diferentes entornos de donde procede la muestra seleccionada, para estudios próximos convendría aumentar el número de participantes y triangular los instrumentos de recogida de datos. Dadas las características y limitaciones de esta investigación, no se pretende, por tanto, generalizar resultados. Aun así, conviene destacar lo significativo de los datos obtenidos. A la información resultante de los objetivos planteados al inicio de esta investigación, es interesante añadir que se han obtenido diferencias relevantes en función de los contextos de referencia. Los resultados señalan que las personas que participan en actividades socio-educativas sienten que se prestan más tiempo a sí mismas y, a su vez, valoran en mayor medida el apoyo que prestan a los demás. Por tanto, cabe destacar el efecto positivo que conlleva para los mayores realizar este tipo de actividades. Como educadoras y desde esta perspectiva, consideramos esencial tener presente los efectos beneficiosos a nivel cognitivo, emocional y social, tratándose de un ámbito de trabajo en el que cabe seguir innovando e investigando. Estudios muy recientes a nivel internacional (Eurydice, 2015), muestran que las personas con menor nivel educativo son menos propensas a buscar actividades de este tipo, por lo que es de gran importancia despertar el interés, adecuar las actividades propuestas y realizar una suficiente divulgación de las mismas. Lo cierto es que el ámbito socio-educativo no queda exento y alejado a las tensiones e intereses económicos de la sociedad, pero convenimos con otros autores en que, paradójicamente sobre lo educativo descansan las grandes esperanzas de la sociedad en la que vivimos (Argüello et al., 2012). De hecho, con este estudio se ha pretendido una revalorización del papel que juegan los mayores en sus familias, subrayando el fuerte capital social que suponen. En efecto, dentro de los distintos tipos de solidaridad intergeneracional, la crisis económica ha reforzado la importancia de la "solidaridad estructural" (forma de convivencia y proximidad) y la solidaridad funcional (prestación de ayuda y cuidados) (Sabater, 2014, 164). Inevitablemente, es preciso destacar cómo la participación en actividades de tipo socioeducativo, junto con el desarrollo de procesos de participación y compromiso social, pueden promover y facilitar una más ajustada autopercepción y un mayor compromiso adecuado a las exigencias de los diferentes entornos en los que se mueve el colectivo diverso y plural que conforman las personas mayores en la actualidad.

\section{Referencias}

Alfageme, A. (2007). The clients and functions of Spanish university programmes for older people: a sociological analysis. Ageing and Society, 27, 343-361.

Andreu, J.M. (2013). España, Europa y el drama político actual. Boletín económico de ICE, 3037, 55-70.

Argüello, A. et al. (2012). Del modelo de desarrollo económico al paradigma del desarrollo humano: una apuesta al papel del arte y las humanidades en el pensamiento de Martha Nussbaum. Revista Complutense de Educación, 23(2), 401-425.

Baker, D., \& Silverstein, M. (2008). Depressive symptoms among grandparents raising grandchildren: the impact of participation in multiple roles. Journal of Intergenerational Relationships, 6, 285-304.

Calero, R. et al. (2009). Cuidador informal de personas mayores dependientes y estrés percibido: intervención psicoeducativa. Scientia, 14(1), 9-19. 
Campo, M. J. (2000). Apoyo informal a las personas mayores y el papel de la mujer cuidadora. Madrid: Centro de Investigaciones Sociológicas.

Cáritas Española (2013). VIII Informe del Observatorio de la Realidad Social: Empobrecimiento y desigualdad Social. El aumento de la fractura social en una sociedad vulnerable que se empobrece. Madrid: Cáritas Española.

Colom, A. (2012, febrero) "La crisis económica española: orígenes y consecuencias: una aproximación crítica". XIII Jornadas de economía crítica. Sevilla.

Cruces, J. (2013). El impacto de la crisis en las condiciones de vida de las Personas Mayores. Colección Informes 56. Madrid: Fundación $1^{\circ}$ de Mayo, Comisiones Obreras.

Cruz Roja (2012). L’impacte de la crisi en les persones grans. Barcelona: Cruz Roja.

Encuentro de sedes del APFA. Evaluación general 2012-2013.

European Commission (2015). Adult Education and Training in Europe: Widening Access to Learning Opportunities. Eurydice Report. Luxembourg: Publications Office of the European Union.

Fernández, I. (2013). Crisis financiera y medios públicos en España. Derecom, 12, 17-30.

Fernández, R. et al. (2013). Active Aging: A Global Goal. Current Gerontology and Geriatrics Research, 1-4.

Fernández, A., García, J.L. y Pérez, G. (2014). Los Programas Universitarios de Mayores y su contribución al aprendizaje a lo largo de la vida. Revista Complutense de Educación, 25(2), 521-540.

Fundación FOESSA (2014). Precariedad y cohesión social. Madrid: FOESSA.

Guerrero, C. (2012). Evaluación de los programas universitarios para mayores: autopercepción de los alumnos. Enlace en red. 22, 24-27.

Guzmán, J.M., Huenchuan, S. y Montes de Oca, V. (2003, julio). Redes de apoyo social de personas mayores: marco teórico conceptual. En Simposio Internacional Viejos y Viejas. Participación, Ciudadanía e Inclusión Social. Santiago de Chile.

Hidalgo, Á., Milán, M. J. y Pérez, S. (2008). Composición y estructura de los hogares sustentados por personas mayores. Universidad de Castilla-La Mancha.

IMSERSO (2007). El apoyo a los cuidadores de familiares dependientes en el hogar: desarrollo del programa "Cómo mantener su bienestar". Colección Estudios. Serie Dependencia, 12006. Madrid: IMSERSO.

IMSERSO (2010). Los Tiempos del Cuidado. Colección Estudios. Serie Dependencia, 12011. Madrid: IMSERSO.

IMSERSO (2012). Las personas mayores en España. Informe 2010. Madrid: IMSERSO.

Instituto Nacional de Estadística (2012a). Boletín informativo del Instituto Nacional de Estadística: Condiciones de vida. Madrid: INE.

Instituto Nacional de Estadística (2012b). Encuesta de Presupuestos Familiares, año 2011. Notas de Prensa. Madrid: INE.

Kirby, J.N. y Sanders, M. (2013). The acceptability of parenting strategies for grandparents providing care to their grandchildren. PreventionScience.

Laparra, M. y Pérez, B. (Coord.). (2012). Crisis y Fractura Social de Europa. Causas y Efectos en España. Barcelona: Fundación "La Caixa".

López, M. T. (2013). Análisis de los comportamientos socioeconómicos de las familias en España (Resumen). Ministerio de Sanidad, Servicios Sociales e Igualdad.

Martínez, L. (2014). Crisis en familia. Síntomas de agotamiento de la solidaridad familiar. VII Informe sobre exclusión y desarrollo social en España. Fundación FOESSA. 
Montero, I., García, J. y Bedmar, M. (2011). Ciudadanía activa y personas mayores. Contribuciones desde un modelo de educación expresiva. Revista Iberoamericana de Educación, 55(5), 1-13.

Montero, I. e Muñoz, I. (2013). Ciudadanos de una sociedad en crisis. Una mirada desde el colectivo de las personas mayores. En S. Torio et al. La crisis social y el Estado de Bienestar. Las respuestas de la Pedagogía Social (518-523). Oviedo: Universidad de Oviedo.

Ortega, M. (2007). El cuidado de los hijos y el género (Tesis doctoral). Málaga: Universidad de Málaga.

Pérez, J., Abellán, A. y Pujol, R. (2013). La generosidad de los mayores españoles. Envejecimiento en-red. http://envejecimiento.csic.es/documentos/blog/La-generosidad-de-los-mayores-espanioles.pdf

Rodríguez, C. (2013). Las familias y los cuidados a las personas mayores dependientes: entre la reciprocidad y la ambivalencia. Cuadernos de Trabajo Social, 26, 349-358.

Sabater, C. (2014). Crisis y condiciones de vida de los mayores en España. Revista Documentación Social, 174, 147169.

Salvetti y Llombart (2015). El papel de los abuelos en la crisis económica. Madrid: Educo.

TFW (2014): Barómetro de la familia, situación y perspectiva. Instituto Internacional de estudios sobre la Familia. Madrid: TFW.

Tobío, C., et al. (2010) El cuidado de las personas, un reto para el siglo XXI. Colección de Estudios Sociales, 28. Barcelona: La Caixa.

Vandell, D.L., et al. (2003). Variations in child care by grandparents during the first three years. Marriage Fam, 65, 375381.

Villar, J.M. (2009). La crisis económica actual. Sus orígenes y características. Medidas para salir de la misma. Los Martes en la Academia (ciclo de conferencias). Madrid: Real Academia de Ingeniería.

Villar, F. (Coord.). (2010). Evaluación de programas universitarios para mayores: Motivaciones, dificultades y contribuciones a la calidad de vida demanda y prestación de cuidados en el seno familiar. Portal Mayores. Madrid: IMSERSO.

Yangua, J. Leturia, M. y Leturia, F. (2000). Apoyo informal y cuidado de las personas mayores dependientes. Papeles del psicólogo, 76, 4. 
ANEXO 1

\begin{tabular}{|l|l|l|l|l|}
\multicolumn{4}{l|}{} & $\begin{array}{l}\text { No de cuestionario: } \\
\text { Fecha: }\end{array}$ \\
\hline $\begin{array}{l}\text { Contexto en el que se recogen los datos } \\
\text { (a rellenar por el encuestador) }\end{array}$ & Residencia & Centro de día & $\begin{array}{l}\text { Aula } \\
\text { Permanente }\end{array}$ & $\begin{array}{l}\text { Asistencia } \\
\text { domiciliaria }\end{array}$ \\
\hline
\end{tabular}

Este cuestionario forma parte de una investigación aprobada por la Universidad de Granada. Tiene como finalidad analizar cómo está influyendo la crisis económica en las personas mayores. El cuestionario es anónimo por lo que le pedimos que conteste con la mayor sinceridad. Muchas gracias por su participación. Por favor, si tiene cualquier duda a la hora de cumplimentarlo, pregunte al encuestador.

Edad: $55-64$ años $\square$

65-74 años

75-84 años

85 años o más

Sexo: Hombre $\square$

Mujer

Lugar en que reside: En mi casa $\square$

En casa de mis hijos $\square$

Otros $\square$ Indique dónde:

Personas con las que convive: Hijos/as $\square$

Pareja

Otros familiares

Personas que no son familiares

Vive solo/a

Indique el número de hijos:

Estado civil: Soltero/a

Casado/a

Viudo/a

Divorciado/a

Nivel de ingresos mensuales: 300 euros o menos

Entre 301 y 549 euros

Entre 550 y 899 euros

Entre 900 y 1199 euros

1200 euros o más

No tiene ingresos

A continuación, marque con una $X$ el tipo de contacto que tiene con su familia (en persona, por teléfono...) y la frecuencia con que mantiene contacto (a diario, cada mes...)

\begin{tabular}{|l|l|l|l|l|}
\hline Tipo de contacto/ frecuencia & Diariamente & Semanalmente & Mensualmente & $\begin{array}{l}\text { Algunas veces a } \\
\text { lo largo del año }\end{array}$ \\
\hline En persona & & & & \\
\hline Por teléfono & & & & \\
\hline Por internet & & & & \\
\hline
\end{tabular}

Indique si participa semanalmente en alguna de estas actividades de ocio:

Hogares y clubes para pensionistas

Hacer ejercicio $\square$

Ir al bar, cafetería

Voluntariado $\square$

Otra actividad. Indique cuál: 
Ahora pasamos a la segunda parte del cuestionario. En caso de que tenga contacto frecuente con su familia lea las siguientes frases y marque con una cruz $(\mathrm{X})$ la respuesta que crea correcta:

¿Presta ayuda y apoyo a las personas de su entorno cuando lo necesitan?

Siempre $\square \quad$ Casi siempre $\square \quad$ A veces $\square \quad$ Nunca $\square$

¿Es importante para usted ayudar y preocuparse por el bienestar de las personas que tiene alrededor?

Muy de acuerdo $\square \quad$ De acuerdo $\square \quad$ En desacuerdo $\square \quad$ Muy en desacuerdo

¿Considera que a partir de la crisis económica ha tenido que prestar más apoyo a su familia?

Si $\square$ No

Si a partir de la crisis económica presta más apoyo a su familia, marque en qué aspectos le presta más apoyo (puede marcar todas las opciones que quiera):

- Cuido de mis nietos diariamente

- Mis hijos (y/o nietos) comen en mi casa a diario

- Colaboro en las tareas del hogar de mis hijos

- Presto apoyo económico de manera regular

- He tenido que hacer un préstamo importante a mis hijos

- Mis hijos todavía no han podido independizarse y viven conmigo

- Mis hijos (y nietos, si es el caso) han vuelto a vivir conmigo por motivos económicos

- Mis hijos residen en una vivienda que es de mi propiedad $\square$

- He avalado a mis hijos en el préstamo que han solicitado

- Soy el sustentador principal de la familia

- Otros apoyos $\square$ Indique cuáles:

Si a partir de la crisis económica presta más apoyo a su familia, indique cómo percibe este cambio (puede marcar todas las opciones que quiera):

- En general, me siento mucho mejor

- Me siento más útil $\square$

- Me siento más valorado/a por mi familia

- Me gustaría poder ayudar más a mi familia

- En ocasiones, siento que mi familia se aprovecha de mi ayuda

- Tengo menos tiempo para mí que antes

- Siento que mi familia no valora mi ayuda lo suficiente

- Me gustaría tener más tiempo para mí

- $\quad$ No me identifico con las opciones anteriores $\square$ Explique aquí cómo percibe este cambio: 